\title{
Práctica deportiva extraescolar y su relación con el autoconcepto y el rendimiento académico
}

\author{
Víctor Marín-De la Fuente1, Mario Alguacil2, Paloma Escamilla- \\ Fajardo $^{3}$

1,2Universidad Católica de Valencia, 3Universidad de Valencia
$\begin{aligned} & \text { Email: } \quad \text { victor.marin6@mail.ucv.es, } \\ & \text { paloma.escamilla@uv.es }\end{aligned}$

RESUMEN: La falta de actividad física en la sociedad es uno de los factores de riesgo más importantes para sufrir enfermedades y una de las causas de muerte más representativas. La sociedad propone alternativas, pero cada vez más sedentarias y es por ello que la práctica deportiva debe potenciarse para ayudar a tener una buena calidad de vida, mejorando a nivel físico, pero también a nivel de autoconcepto, rendimiento académico y a nivel social, por lo que es una buena herramienta para propiciar el desarrollo integral de las personas. En este estudio se ha analizado el autoconcepto, el rendimiento académico y la práctica deportiva extraescolar de los alumnos, con la intención de comprobar si el hecho de realizar práctica extraescolar o no, supone cambios en los niveles de autoconcepto y rendimiento académico, así como comprobar si existe significación en la relación entre el autoconcepto de los alumnos y su rendimiento académico. Los resultados muestran que los alumnos que practican deporte extraescolar obtienen mejores medias de autoconcepto y rendimiento académico, de la misma manera que las mujeres obtienen medias más altas que los hombres, aunque solamente se puede decir que existan diferencias significativas en los valores de autoconcepto en función del curso al que pertenecen.

PALABRAS CLAVE: Autoconcepto, autoestima, rendimiento académico, práctica extraescolar.

\section{Extracurricular sports practice and its relationship to self-concept and academic performance}

ABSTRACT: The lack of physical activity in society is one of the most important risk factors for suffering diseases and one of the most representative causes of death. Society proposes alternatives, but increasingly sedentary and it is for this reason that the practice of sport should be promoted to help to have a good quality of life, improving at a physical level, but also at a level of self-concept, academic performance and social level, so it is a good tool to promote the integral development of people. In this study, the self-concept, academic performance and extracurricular sports practice of students have been analyzed, with the intention of verifying if the fact of doing extracurricular practice or not implies changes in the levels of self-concept and academic performance, as well as verifying if there is significance in the relationship between the self-concept of students and their academic performance. The results show that students who 
practice extracurricular sports obtain better averages of self-concept and academic performance, in the same way that women obtain higher averages than men, although it can only be said that there are significant differences in self-concept values depending on the course to which they belong.

KEY WORDS: Self-concept, Self-esteem, academic performance, extracurricular practice 


\section{INTRODUCCIÓN}

El estudio de la autoestima, el autoconcepto y su relación con la práctica de actividad física y el rendimiento académico, es un tema que ha sido ampliamente estudiado a lo largo del tiempo por numerosos autores. En algunas ocasiones, estos estudios han relacionado positivamente la actividad física con la autoestima, el autoconcepto y el rendimiento académico mientras que en otros casos no ha sido así, lo que motiva a comprobar este aspecto y el resultado que podría obtenerse analizando un centro educativo de interés. Este ámbito de estudio es un ámbito que incluye temas de estudio de grandes dimensiones, donde es muy importante la investigación ya que la información que se pueda extraer en esos estudios puede influir significativamente en un mejor desarrollo integral de la persona, no solo centrado en un aspecto concreto sino tratando de mejorar todo el conjunto del individuo. Por otro lado, el análisis del rendimiento académico es fundamental ya que supone en gran medida la adaptación y el éxito en la sociedad y en ambos casos, tanto en la autoestima y autoconcepto, como en el rendimiento académico, la práctica de actividad física debe aparecer como herramienta de mejora, ya que existen beneficios probados en relación a estos aspectos.

\section{MARCO TEÓRICO}

\section{Desarrollo cognitivo}

El aprendizaje otorga cierta capacidad de autocontrolar la conducta humana y permite la adaptación de la mejor manera posible al entorno. Para ello, es necesario tanto adquirir conductas nuevas como inhibir las que no lo son, por lo que esto supone un cambio continuo en el comportamiento (Ruiz, 2010). En cuanto al proceso de aprendizaje, se entiende por desarrollo cognitivo el estudio de la evolución de los procesos psicológicos humanos, desde los más simples hasta los más complejos, entendiendo como simples la percepción, atención o aprendizaje y como más complejos el pensamiento, la imaginación, el lenguaje o el razonamiento (Enesco, 2001). Para Piaget, el equilibrio es el responsable del desarrollo cognitivo y se da como resultado de un proceso de asimilación/acomodación que le permite adaptarse al medio, mientras que, para Vygotsky, el desarrollo cognitivo se da por las relaciones sociales en los procesos mentales superiores y viene determinada por la internalización de las herramientas y la interacción con el medio social (Moreira, 1997, citado en Martínez, Arrieta y Meleán, 2012).

En cuanto a la visión del desarrollo cognitivo por parte de los investigadores clásicos, según se explica en el estudio de Vielma y Salas (2000), Piaget propone un paradigma en el que el ser humano se desarrolla debido a la maduración orgánica y a su historia personal, que empieza como una unidad individual que con el tiempo se transformará en social debido al contacto con otras personas del entorno. Por otro lado, Vygotsky nos habla de un paradigma donde el niño se desarrolla por su maduración orgánica pero fundamentalmente por la cultura y el entorno donde se encuentra inmerso, ya que entiende que los procesos mentales solo pueden ser entendidos si se entienden los procesos sociales y culturales del contexto donde vive. Posteriormente, Bandura propone que el desarrollo cognitivo humano se lleva a cabo por el aprendizaje en contextos sociales a través de modelos reales y simbólicos, y finalmente, Bruner (1990), entiende que es un paradigma que se produce en la mente, construyendo modelos 
mentales influenciados por el proceso de almacenamiento de estos y las conclusiones deducidas por parte del que aprende.

Teoría de la mente

En cuanto a la teoría de la mente, es definida como una "habilidad psíquica que poseemos para representar en nuestra mente los estados mentales de otro para a través de ella poder explicar y predecir su conducta" (Téllez, 2006, p.11), por tanto, se refiere a la habilidad para comprender y predecir la conducta de otras personas, sus conocimientos, sus intenciones, sus emociones y sus creencias (Tirapu, Pérez, Erekatxo y Pelegrín, 2007), surgiendo esta teoría de las teorías de desarrollo cognitivo comentadas anteriormente (Téllez, 2006). Aunque este proceso va más allá, ya que no sólo se interpretan las acciones de los demás, sino que también se les puedes atribuir intencionalidades (García, González y Maestú, 2011). Esta idea también se traduce en las emociones, puesto que estos procesos también permiten empatizar con otras personas y modificar la conducta en función de la comprensión de las emociones de los demás (García, 2008).

\section{Desarrollo cognitivo y deporte}

En la relación entre desarrollo cognitivo y deporte, no se puede olvidar que, gracias a la capacidad motriz, es posible la adaptación al medio para evolucionar. El desarrollo cognitivo desde temprana edad está íntimamente relacionado con el movimiento, ya que a través de él se obtienen las primeras experiencias sensoriales por las que el niño explora, relaciona y controla su ambiente, lo que le permite empezar a construir sus esquemas mentales.

Gracias a la neurociencia y la neuropsicología, se ha podido demostrar que con la actividad física se realiza una regeneración y una activación de conexiones neuronales que contribuye al aprendizaje motor y también al desarrollo cognitivo (Loayza, 2016). Existen estudios que comprueban que los ejercicios de coordinación mejoran las capacidades atencionales y otras funciones cognitivas. Smith (2006) basa sus estudios en el principio de que determinados ejercicios físicos, como por ejemplo la ejecución de movimientos contralaterales también conocidos como patrones cruzados, estimulan el trabajo conjunto de los dos hemisferios cerebrales favoreciendo la cognición. Es por todo esto por lo que "cabe destacar el valor y la importancia, subestimada por la comunidad educativa, de la Educación Física en los programas escolares" (Pinto, 2011, p. 176). Además de la práctica de actividad física reglada, la realización de deporte extraescolar ha demostrado ser una buena opción, ya que continúa favoreciendo este tipo de mejoras y hace que mejore el funcionamiento del cerebro, la concentración, la autoestima y se obtenga un mejor comportamiento, factores que propician una mejora en los procesos de aprendizaje (Padilla, Aguilar y Fernández, 2016). En este sentido, se explica que el mayor rendimiento académico se produce en los niños que practican actividad física extraescolar moderada, es decir, entre 2 y 5 horas semanales, produciéndose el peor rendimiento en los niños que realizan un exceso de práctica (Cladellas, Clariana, Gotzens, Badia y Dezcallar, 2015). 


\section{Autoestima y Autoconcepto}

Según Montoya y Sol (2001) y González-Arratia (2011), la autoestima es la apreciación que cada persona hace de sí misma mediante un proceso valorativoacumulativo, basado en la experiencia y con un enfoque general que se ve influenciado por el conjunto de estados anímicos que aparecen a lo largo de periodos de tiempo relativamente continuos y duraderos (Silva y Mejía, 2015). En las personas con baja autoestima, el sentirse inadecuado, inadaptado, inseguro, incapaz y sin valor, son mucho más frecuentes debido a sus experiencias negativas en la consecución de logros o necesidades personales (Mejía, Pastrana y Mejía, 2011).

El autoconcepto, por su parte, es la idea que cada persona tiene de sí misma y de su realidad en función de las experiencias. Por último, aparece el concepto de autorrealización, que es la consecución de logros que provocan sentimientos de plenitud, pudiendo tener que ver con los demás o con uno mismo (Silva y Mejía, 2015). Simplemente el sentimiento de pertenencia a un grupo y la aceptación de este hacia la persona afectará de forma positiva a la autoestima, lo que no se relaciona de forma directa con el tipo de comportamientos o la forma de pensar que se puedan desarrollar con la pertenencia a dicho grupo (Álvarez, Sandoval y Velásquez, 2007).

Por ello, será bueno que el adolescente adquiera valores como la autodecisión, la autonomía, la autodisciplina, el autocontrol, la autosuficiencia, la autoaceptación y la autorrealización para afrontar la vida con una autoestima positiva. Conseguir esto será de vital importancia puesto que esta influirá de manera significativa en los sentimientos del niño, en su aprendizaje, en su pensamiento en sus relaciones, en su comportamiento y en su propia consideración (Mejía et al., 2011). El autoconcepto implica los aspectos cognitivos o de pensamiento, relacionados con la autoimagen y en general, se hace referencia a la totalidad de un complejo organizado. Un sistema dinámico de creencias aprendidas, actitudes y opiniones que cada persona tiene como valederas acerca de su existencia personal (Villasmil, 2010). A diferencia de la anteriormente comentada autoestima en la que prevalecen principalmente las dimensiones valorativa y afectiva, en el autoconcepto prevalece la cognitiva, la percepción y la imagen que cada uno tiene de sí mismo. Este concepto tiene una realidad muy general pero dentro se distinguen otros más específicos como pueden ser el físico, académico, social, personal y emocional (Roa, 2013).

El niño se introduce en la etapa escolar y adolescencia, punto álgido en el desarrollo del autoconcepto, donde empieza el desarrollo tanto cognitivo como físico que serán determinantes para su integración entre iguales. Además, esta maduración le dotará de libertad para conceptualizarse con la sociedad lo que conllevará una reestructuración del autoconcepto (Cazalla y Molero, 2013).

En relación con la práctica deportiva, la posesión de una buena autoestima dota de capacidad para afrontar de forma más realista las actividades deportivas, lo que ayuda a lograr éxitos y a encajar los fracasos (Hurtado, 2014). Además, la practica regular de ejercicio aeróbico y moderado contribuye a la prevención de enfermedades mentales habiéndose demostrado que las personas que abandonan las prácticas deportivas muestran síntomas de negatividad y tienen más posibilidades de padecer conductas depresivas (Candel, Olmedilla y Blas, 2008). En otras etapas, como la tercera edad, el ejercicio físico también tiene beneficios. Como expresan García, Marín y 
Bohórquez (2012) las personas mayores que realizan actividad física de forma regular muestran una mayor aceptación de la etapa de la vejez, sintiéndose bien consigo mismo y con los demás. Esta práctica deportiva supone una contribución para la mejora del autoconcepto físico, incidiendo así en los dominios académico, social, emocional y familiar (Murgui, García y García, 2016). Finalmente, hay estudios que no relacionan de forma positiva la realización de actividad física con la mejora de la autoestima, ya que entienden que la mejora de la autoestima física no está ligada con la mejora de la autoestima global, siendo un concepto muy estable a lo largo de la vida, en el que la actividad física no puede ser muy determinante (Herrera, Pablos, Chiva y Pablos, 2016). Esta disonancia de opiniones es la que justifica y motiva para la realización de este estudio en el caso de un instituto de la ciudad de Sagunto.

\section{OBJETIVOS}

El objetivo del trabajo es analizar la relación de la práctica de actividad física extraescolar con el nivel de autoconcepto y el rendimiento académico de los alumnos.

Como objetivos específicos se pretende saber si existe una correlación significativa entre el nivel de autoconcepto académico y el rendimiento académico y si los niveles de autoconcepto de los alumnos difieren significativamente en función de las variables de género y curso.

\section{MÉTODO}

Muestra

En lo referente a la muestra, la población objeto de estudio son los alumnos de la etapa secundaria obligatoria (E.S.O) de un instituto situado en el municipio de Sagunto. La muestra consta de 80 alumnos, de los 160 aproximadamente que tiene el centro en dicha etapa. Del total de encuestados, el $50 \%$ son chicas $(n=40)$ y el $50 \%$ chicos $(n=$ 40) siendo 20 alumnos de cada curso en una proporción del $50 \%$ en base al género en cada caso. Además del género y el curso, se ha tenido en cuenta si han realizado o no deporte extraescolar.

Instrumento

El instrumento utilizado para la realización de este trabajo ha sido el cuestionario de autoconcepto AF5 de García y Musitu (2001). Dicho instrumento se compone de 30 elementos con los que se evalúan 5 dimensiones diferentes del autoconcepto presentes en el sujeto: en primer lugar, el aspecto académico-laboral, seguido del social, emocional, familiar y, por último, del aspecto físico. Cada una de estas dimensiones se evalúa a través de diferentes ítems que aportan información sobre cada aspecto concreto.

Procedimiento

En cuanto al procedimiento seguido, se solicitó permiso al centro para poder realizar el proyecto. Posteriormente, se realizó una hoja de consentimiento informado que se les entregó a los alumnos para que fuese firmada por los padres o tutores de los 
niños y, una vez se tenía el permiso de todas las partes, se procedió a la recogida de datos mediante un cuestionario anónimo.

Análisis estadístico

Para extraer los resultados se ha utilizado el programa estadístico SPSS en su versión 23. En primer lugar, se ha llevado a cabo una prueba de normalidad, para comprobar si las variables usadas siguen una distribución normal y, por tanto, si podemos usar estadística paramétrica. Una vez confirmado este punto, se han realizado pruebas T para muestras independientes, con la intención de observar si las medias de los niveles de autoconcepto y de rendimiento académico son significativamente diferentes en función de si practican o no deporte, y también si los valores de autoconcepto general se mostraban diferentes en base al género. También se ha llevado a cabo una correlación para identificar si la variable de autoconcepto académico y la del rendimiento académico estaban significativamente relacionadas. Por último, se ha realizado la prueba ANOVA de un factor para comprobar si los niveles de autoconcepto general eran significativamente diferentes en los cuatro cursos analizados.

\section{RESULTADOS}

En primer lugar, se ha realizado un análisis de normalidad de los datos, para determinar la idoneidad de las pruebas estadísticas que se pretenden llevar a cabo en el estudio. Una vez realizada, la prueba resultó no significativa $(p>.05)$ en las variables a utilizar, por lo que se entiende que los datos siguen una distribución normal y que, por tanto, se puede proceder a utilizar pruebas de estadística paramétrica, como es el caso de las pruebas $T$ y el ANOVA de un factor.

Posteriormente, se ha llevado a cabo la comparación de los niveles de autoconcepto entre las personas que realizan actividad física y las que no. Como se puede apreciar en la Tabla 1, la puntuación media que se ha obtenido respecto a la variable de autoconcepto ha resultado ser mayor en los estudiantes que practican deporte extraescolar con un valor medio de $57.21( \pm 19.15)$ respecto a los que no lo hacen (53.82 \pm 19.15$)$. Sin embargo, tras realizar la comparación mediante una prueba $\mathrm{T}$, el resultado ha sido no significativo ( $p>.05$ ), por lo que se puede afirmar que no existe una diferencia significativa respecto a los valores de autoconcepto entre las personas que realizan actividad física extraescolar y las que no.

Tabla 1. Descriptivos autoconcepto en base a la práctica de actividad física

\begin{tabular}{cccc}
\hline & $\begin{array}{c}\text { Deporte } \\
\text { Extraescolar }\end{array}$ & X & DT \\
\hline Autoconcepto & Sí practica & 57.21 & 19.15 \\
general & No practica & 53.82 & 17.73 \\
\hline
\end{tabular}

Posteriormente, se realizó de nuevo el mismo procedimiento, pero en este caso con la intención de analizar una comparación entre la nota media de los alumnos en base a si dichos estudiantes practican actividad física extraescolar o no. La nota media de las personas que practican deporte es superior a los que no practican (ver Tabla 2), con valores de $6.34( \pm 1.32)$ y $5.85( \pm 1.54)$, respectivamente. Sin embargo, tras la 
realización de la comparación de medias, el resultado no ha mostrado resultados estadísticamente significativos ( $p>.05$ ), por lo que no se puede confirmar que el rendimiento académico sea significativamente diferente entre los que practican actividad física y los que no.

Tabla 2. Descriptivos rendimiento académico en base a la práctica de actividad física

\begin{tabular}{cccc}
\hline & Deporte extraescolar & X & DT \\
\hline \multirow{2}{*}{ Nota media } & Si Practica & 6.34 & 1.32 \\
& No Practica & 5.85 & 1.54 \\
\hline
\end{tabular}

Seguidamente, tras las diferentes comparaciones llevadas a cabo, se quería comprobar si podía existir una correlación significativa entre la variable relacionada con el autoconcepto académico y el rendimiento académico de los estudiantes. Una vez realizada la prueba de correlación y obtenidos los resultados (ver Tabla 3) con el valor de correlación de Pearson, observamos que dicha correlación no es significativa ( $p>.05)$ $y$, por tanto, no es posible afirmar que exista una correlación estadísticamente significativa entre el autoconcepto académico y el rendimiento académico.

Tabla 3. Correlación entre autoconcepto académico y nota media

\begin{tabular}{cc}
\hline & Autoconcepto \\
\hline Nota media Correlación de Pearson & .21 \\
\hline Nota: ${ }^{*} p<.05$ &
\end{tabular}

En cuanto a la comparación del autoconcepto en base al género (ver Tabla 4), como se puede apreciar las mujeres tienen mejor media que los hombres ( $57.49 \pm 18.98$; $53.53 \pm 17.82$, respectivamente). Tras la realización de la comparación de medias ( $p>$.05) se puede afirmar que hombres y mujeres no obtienen puntuaciones significativamente diferentes en cuanto a su autoconcepto.

Tabla 4. Comparación de los niveles de autoconcepto por género

\begin{tabular}{lccc}
\hline & & $\mathrm{X}$ & $\mathrm{DT}$ \\
\hline \multirow{2}{*}{ Autoconcepto general } & Hombre & 53.53 & 17.85 \\
& Mujer & 57.49 & 18.98 \\
\hline
\end{tabular}

Para finalizar, se ha realizado una comparativa de los niveles de autoconcepto en base al curso al que pertenecen los alumnos. En la Tabla 5 se puede apreciar que los mejores datos respecto al autoconcepto por cursos se encuentran en segundo, con una media de $66.59( \pm 12.15)$ seguidos por primero con una media de $58.17( \pm 16.16)$, tercero con una media de $56.08( \pm 17.02)$ y, finalmente, cuarto con una media de $41.20( \pm 18.95)$.

Tabla 5. Descriptivos de los niveles de autoconcepto por curso

\begin{tabular}{ccc}
\hline & X & DT \\
\hline 1 NO & 58.17 & 16.16 \\
2을 ESO & 66.59 & 12.15
\end{tabular}




\begin{tabular}{lrr}
$3^{\circ}$ ESO & 56.08 & 17.02 \\
4 NO & 41.20 & 18.95 \\
Total & 55.51 & 18.42 \\
\hline
\end{tabular}

Con el fin de comprobar si existen diferencias estadísticamente significativas entre los grupos planteados, se ha realizado una prueba ANOVA ( $p<.001$ ) en la que como se puede comprobar en la Tabla 6 , se ha encontrado que las diferencias son estadísticamente significativas entre los grupos de los cursos de primero y cuarto $(p<.01)$ y también entre los cursos de segundo y tercero $(p<.05)$.

Tabla 6. Prueba Post Hoc comparación entre cursos

\begin{tabular}{|c|c|c|c|c|c|}
\hline \multirow{3}{*}{$1^{\circ} \mathrm{ESO}$} & $2^{\circ}$ ESO & .106 & \multirow[b]{2}{*}{ 3 ESO } & 1 ESO & .686 \\
\hline & $3^{\circ} \mathrm{ESO}$ & .686 & & $2^{\circ} \mathrm{ESO}$ & .044 \\
\hline & $4^{\circ} \mathrm{ESO}$ & .001 & & $4^{\circ} \mathrm{ESO}$ & .005 \\
\hline \multirow{3}{*}{$2^{\circ}$ ESO } & $1^{\circ} \mathrm{ESO}$ & .106 & \multirow{3}{*}{$4^{\circ}$ ESO } & $1^{\circ} \mathrm{ESO}$ & .001 \\
\hline & $3^{\circ} \mathrm{ESO}$ & .044 & & $2^{\circ} \mathrm{ESO}$ & .000 \\
\hline & $4^{\circ} \mathrm{ESO}$ & .000 & & $3^{\circ} \mathrm{ESO}$ & .00 \\
\hline
\end{tabular}

\section{DISCUSIÓN}

El presente estudio ha tenido como objetivo analizar la influencia de la actividad física y el deporte extraescolar sobre el autoconcepto y el rendimiento académico. En líneas generales, los resultados no llegan a completarse como sí ocurre en muchos de los estudios relacionados con este tema, en los que se establece que la práctica deportiva influye positivamente sobre la autoestima y el rendimiento académico. En este sentido, autores como Guillén y Nieri (2009) y Moreno, Cervelló y Moreno (2008) defienden que las personas que practican deporte tienen una autoestima más elevada que los que no y, en esta línea, Murgui et al. (2016) también establecen que las personas que practican deporte obtienen mayores puntuaciones en todas las dimensiones del autoconcepto, lo que se encuentra de acuerdo con los resultados obtenidos por otros autores como Ramos, Rivera, Moreno y Jiménez (2012) o Jiménez, Zagalaz, Molero, Pulido y Ruiz (2013). Sin embargo, en el caso del presente estudio, a pesar de que los alumnos que practican deporte extraescolar obtienen mejores puntuaciones en cuanto a autoconcepto y rendimiento académico, tras realizar la comparación estadística no se puede afirmar que esa diferencia sea significativa. Estos resultados van en la línea de otros trabajos en los que el resultado ha indicado que no existe significación en las relaciones propuestas (Blasco, 1997; Fox, 2000; Herrera et al. 2016; Molina, Castillo y Pablos, 2007).

Por otro lado, se pretendía analizar la relación entre autoconcepto y rendimiento académico y el papel que podía jugar la práctica de actividad física extraescolar en ambos, dado el interés que ha suscitado la temática en la literatura científica al respecto (Álvarez et al. 2017; Huang, 2011; Skaalvik, \& Skaalvik, 2013). En este sentido, en cuanto al rendimiento académico y la práctica deportiva, encontramos estudios como el de Cladellas et al. (2015) o el de Martín (2014) que revelan que las actividades físicodeportivas influyen significativamente en el rendimiento de los niños de primaria. González y Portolés (2014) siguen esta misma línea, y en su contribución en la que 
analizan la relación de la actividad extraescolar con la motivación, el rendimiento académico y las conductas asociadas a la salud, encuentran diferencias en los distintos índices motivacionales y en algunas asignaturas como lengua y literatura y educación física. Esta conclusión concuerda con otros estudios en los que los alumnos que practican actividad física obtienen significativamente más nota que los que no (Linder, 2002; Ramírez, Vinaccia y Suarez, 2004). Por su parte, en el estudio de Herrero-Torres, Al-Lal y Mohamed (2017) se obtiene que las correlaciones entre las calificaciones de las asignaturas evaluadas y el autoconcepto son positivas, y dentro de ese autoconcepto, en concreto aparecen en el caso del autoconcepto académico, social y familiar. Esto muestra el papel importante de la familia y el entorno, un papel que es importante en toda la etapa educativa y donde en los últimos años de primaria y primeros años de secundaria la aceptación social juega un rol decisivo (Madariaga y Goñi, 2009). Sin embargo, en el caso de la presente investigación, a pesar de que las puntuaciones medias son más altas en aquellos alumnos que practican actividad física extraescolar, no se ha encontrado significación en dicho aspecto, algo que por otro lado no ha sido inusual, encontrando aportaciones como la de Dimas (2006), Barría y Manríquez (2011) o Pamos (2016) con resultados similares.

Finalmente, respecto a la posible correlación entre el autoconcepto académico y la nota media, existen estudios que confirman dicha relación significativa, como el de Cruz y Quinones (2012) o el de Rabell (2012) entendiendo que un buen autoconcepto influye en la motivación y en consecuencia en el rendimiento académico, donde aspectos como la atención, la motivación y el cociente intelectual pueden ser también influyentes (Hernández, San Miguel y Rodríguez, 2011). Siguiendo esta línea, pero de forma más reciente, encontramos estudios como el de Chacón-Borrego et al. (2017) en el que se analizan los distintos autoconceptos y su relación con la práctica de actividad física en una muestra de jugadoras de baloncesto extraescolar. Los resultados indican que solamente el autoconcepto académico muestra relación significativa con la práctica de actividad extraescolar. Sin embargo, al contrario que los ejemplos citados anteriormente, en la presente investigación la correlación entre autoconcepto académico y rendimiento académico no ha resultado significativa, por lo que no podemos soportar dichas conclusiones.

\section{CONCLUSIONES}

Como conclusiones del estudio, vemos en primer lugar que las personas que practican deporte tienen de media un mejor autoconcepto que las que no practican, aunque al llevar a cabo esta comparación de medias no encontramos diferencias estadísticamente significativas. En segundo lugar, en lo que respecta al rendimiento académico, vemos que en nuestro caso las personas que practican deporte y las que no practican no obtienen valores estadísticamente significativos, en cuanto al rendimiento académico, por lo que no podemos afirmar que la práctica haya sido determinante. Tampoco hemos obtenido significación en la correlación entre el autoconcepto académico del alumno y su nota media, por lo que no se puede afirmar que los valores de autoconcepto académico/profesional tengan una vinculación con la nota media obtenida. En lo que respecta a la comparación de autoconcepto general en base al género, no se han obtenido diferencias estadísticamente significativas entre chicos y chicas, mientras que en la comparación por cursos sí que aparecen, concretamente entre los cursos de primero y cuarto y entre segundo y tercero. 


\section{LIMITACIONES Y LINEAS FUTURAS}

En primer lugar, encontramos la limitación de la muestra, ya que un número mayor de encuestas quizás habría arrojado resultados diferentes o al menos más extrapolables a la población objetivo. También pensamos que puede influir en los resultados de la encuesta el hecho de que se haya contestado cerca de los compañeros, lo que puede hacer que ciertos alumnos tiendan a fijarse en las respuestas de sus compañeros. Como futuras líneas de investigación, creemos que es importante seguir investigando acerca de la influencia que tiene el deporte en el desarrollo integral del niño y demostrando la importancia de esta en la educación. Para ello, se podría realizar un trabajo en el que se evaluara tanto el componente cognitivo mediante las inteligencias múltiples, el componente emocional y el social y el sentimiento de pertenencia grupal, todo ello relacionado con la práctica de actividad física. Además, sería interesante analizar comparaciones, pero teniendo en cuenta los distintos tipos de autoconcepto, para concretar más los resultados.

\section{REFERENCIAS}

Álvarez, A., Sandoval, G. y Velásquez, S. (2007). Autoestima en los (as) alumnos (as) de los 1ํㅡㄹ medios de los liceos con alto índice de vulnerabilidad escolar (I.V.E) de la cuidad de Valdivia. Chile: Universidad Austral de Chile.

Álvarez, A., Suárez, N., Tuero, E., Núñez, J. C., Valle, A., \& Regueiro, B. (2015). Implicación familiar, autoconcepto del adolescente y rendimiento académico. European Journal of Investigation in Health, Psychology and Education, 5(3), 293311.

Barría, P. y Manríquez, M. (2011). Relación entre actividad física regular y rendimiento académico en seleccionados universitarios de deportes colectivos en la universidad austral de chile. Valdivia: Universidad Austral de Chile.

Blasco, M.P. (1997). Beneficios psicológicos de la práctica deportiva en estudiantes universitarios valencianos. Tesis doctoral no publicada. Universidad de Valencia.

Candel, N., Olmedilla, A. y Blas, A. (2008). Relaciones entre la práctica de actividad física y el autoconcepto, la ansiedad y la depresión en chicas adolescentes. Cuadernos de psicología del deporte, 8(1), 61-77.

Cazalla, N. y Molero, D. (2013). Revisión teórica sobre el autoconcepto y su importancia en el autoconcepto. Revista Electrónica de Investigación y Docencia, 1(10), 4364.

Chacón-Borrego, F., Ruz, R. P., Clements, S. Y., Prats, S. B., González, M. D. M. C. y Ortega, F. Z. (2017). Relación entre el rendimiento académico y autoconcepto en jugadoras de baloncesto de categoría cadete en competición nacional extraescolar. Sportk: revista euroamericana de ciencias del deporte, 6(2), 75-80.

Cladellas, R., Clariana, M., Gotzens, C., Badía, M. y Decazllar, T. (2015). Patrones de descanso, actividades físico-deportivas extraescolares y rendimiento académico en niños y niñas de primaria. Revista de Psicología del Deporte, 24(1), 53-59.

Cruz, F. y Quinones, A. (2012). Autoestima y rendimiento académico en estudiantes de enfermería de Poza Rica, Veracruz, México. Veracruz: Universidad de Antioquía.

Dimas, J. L. (2006). Relación entre actividad física y el rendimiento académico en estudiantes de la facultad de ciencias químicas de la UANL. San Nicolás de los Garza: Universidad Autónoma de Nuevo León.

Enesco, I. (2001). Psicología del desarrollo. Universidad Complutense de Madrid: Madrid. 
Fox, K.R. (2000). The effects of exercise on self-perceptions and self-esteem. En S. Biddle, K.R. Fox y S.H. Boutcher (Eds.), Physical activity and psychological wellbeing (pp. 88-117). New York: Routledge.

García, A. J., Marín, M. y Bohórquez, M. R. (2012). Autoestima como variable psicosocial predictora de la actividad física en personas mayores. Revista de Psicología del Deporte, 21(1), 195-200.

García, F. y Musitu, G. (2001). Autoconcepto Forma 5. Tea: Madrid.

García, E. (2008). Neuropsicología y educación. De las neuronas espejo a la teoría de la mente. Revista de psicología y educación, 1(3), 69-90.

García, E., González, J. y Maestú, F. (2011). Neuronas espejo y teoría de la mente en la explicación de la empatía. Ansiedad y estrés, 17(2), 265-279.

Gillén, F. y Nieri, D. (2009). Autoestima y competitividad en una selección juvenil peruana de surf. Revista Iberoamericana de Psicología del ejercicio y el Deporte, 4(2), 253-269.

González-Arratia, L. F. N. I. (2011). Resiliencia y Personalidad en niños y adolescentes. Cómo desarrollarse en tiempos de crisis.

González, J., \& Portolés, A. (2014). Actividad física extraescolar: relaciones con la motivación educativa, rendimiento académico y conductas asociadas a la salud. Revista iberoamericana de psicología del ejercicio y el deporte, 9(1), 51-65.

Herrera, E., Pablos, A., Chiva, Ó. y Pablos, C. (2016). Efectos de un programa integral de ejercicio físico sobre la condición física, la autoestima y el grado de diversión en adultos mayores. Ágora para la educación física y el deporte, 18(2), 167-183.

Huang, C. (2011). Self-concept and academic achievement: A meta-analysis of longitudinal relations. Journal of School Psychology, 49(5), 505-528. doi:10.1016/j.jsp.2011.07.001

Hurtado, S. G. (2014). Nivel de autoestima y éxito deportivo en jóvenes que practican taekwondo (Estudio realizado con deportistas en área de competencias). Universidad Rafael Landivar: Quetzaltenango.

Jiménez-Moral, J. A., Zagalaz, M. L., Molero, D., Pulido-Martos, M. y Ruiz, J. R. (2013). Capacidad aeróbica, felicidad y satisfacción con la vida en adolescentes españoles. Revista de Psicología del Deporte, 22(2), 429-436.

Linder, K. (2002). The Physical Activity Participation Academic Performance Relationship Revisited: Perceived and Actual Performance and the Effect of Banding (Academic Tracking). Pediatric Exercise Science, 14, 155-170.

Loayza, R. (2016). La preparación física y sus beneficios en el desarrollo corporal y cognitivo en los jóvenes de 14 a 16 años. Universidad técnica de Machala: Machala.

Madariaga, J. M., \& Goñi, A. (2009). El desarrollo psicosocial. Revista de Psicodidáctica, $14,95-118$

Martín, A. (2014). Rendimiento académico y actividad física en adolescentes. Un estudio en $2^{\circ}$ ciclo de la ESO del I.E.S Pando de Oviedo. Universidad de Oviedo: Oviedo.

Martínez, R., Arrieta, X. y Meleán, R. (2012). Desarrollo cognitivo conceptual y características de aprendizaje de estudiantes universitarios. Omnia, 18(3), 35-48.

Mejía, A., Pastrana, J. D. y Mejía, J. M. (2011). La autoestima, factor fundamental para el desarrollo de la autonomía personal y profesional. Barcelona: XII Congreso Internacional de Teoría de la Educación.

Molina, J., Castillo, I. y Pablos, C. (2007). Bienestar psicológico y práctica deportiva en universitarios. European Journal of Human Movement, 1(18), 79-91.

Montoya, M. y Sol, C. E. (2001). Autoestima. Estrategias para vivir mejor con técnicas de PNL y desarrollo humano. México, Pax. 
Moreno, J. A., Cervelló, E. y Moreno, R. (2008). Importancia de la práctica físicodeportiva y del género en el autoconcepto físico de los 9 a los 23 años. International Journal of Clinical and Health Psychology, 8(1), 171-183.

Murgui, S., García, C. y García, Á. (2016). Efecto de la práctica deportiva en la relación entre las habilidades motoras, el autoconcepto físico y el autoconcepto multidimensional. Revista de Psicología del Deporte, 25(1), 19-25.

Padilla, D., Aguilar, J. M. y Fernández, J. M. (2016). La actividad física y deportiva como medio para la reducción de los niveles de deterioro cognitivo en las personas mayores. International Journal of Developmental and educational Psychology, 465- 474.

Pamos, A. (2016). Relación entre la actividad física y el rendimiento académico en educación primaria. Universidad de Jaén: Jaén.

Pinto, M. F. (2011). El impacto del deporte en la promoción del desarrollo cognitivo de los niños. Su relevancia para la inclusión de la actividad física en programas de promoción de la salud. Universidad de Buenos Aires: Buenos Aires.

Rabell, M. (2012). Autoestima y rendimiento académico: un estudio aplicado al aula de educación primaria. Universidad de la Rioja: Barcelona.

Ramírez, W., Vinaccia, S., y Suárez, G. R. (2004). El impacto de la actividad física y el deporte sobre la salud, la cognición, la socialización y el rendimiento académico: una revisión teórica. Revista de estudios sociales, 18, 67-75.

Ramos, P., Rivera, F., Moreno, C. y Jiménez-Iglesias, A. (2012). Análisis de clúster de la actividad física y las conductas sedentarias de los adolescentes españoles, correlación con la salud biopsicosocial. Revista de Psicología del Deporte, 21(1), 99-106.

Roa, A. (2013). La educación emocional, el autoconcepto, la autoestima y su importancia en la infancia. Revista Edetania, 241-257.

Ruiz, Y. M. (2010). Aprendizaje vicario: Implicaciones educativas en el aula. Revista digital para profesionales de la enseñanza, 1-6.

Silva, I. y Mejía, O. (2015). Autoestima, adolescencia y pedagogía. Revista electrónica educare, 19(1), 241-256.

Skaalvik, E. M., \& Skjaalvik, S. (2013). School goal structure: Associations with students' perceptions of their teachers as emotionally supportive, academic self-concept, intrinsic motivation, effort, and help seeking behavior. International Journal of Educational Research, 61, 5-14. doi:10.1016/j.ijer.2013.03.007

Smith, A. (2006). Speech motor development: Integrating muscles, movements, and linguistic units. Journal of communication disorders, 39(5), 331-349.

Téllez, J. (2006). Teoría de la mente: Evolución, ontogenia, neurobiología y psicopatología. Avances en psiquiatría biológica, 1-22.

Tirapu, J., Pérez, G., Erekatxo, M. y Pelegrín, C. (2007). ¿Qué es la teoría de la mente? Revista de Neurología, 44(8), 479-489.

Vielma, E. y Salas, M. L. (2000). Aportes de las teorías de Vygotsky, Piaget, Bandura y Bruner. Paralelismo en sus posiciones en relación con el desarrollo. Educere, 3(9), 30-37.

Villasmil, J. (2010). El autoconcepto académico en estudiantes universitarios resilientes de alto rendimiento: un estudio de casos. Universidad de los Andes. 